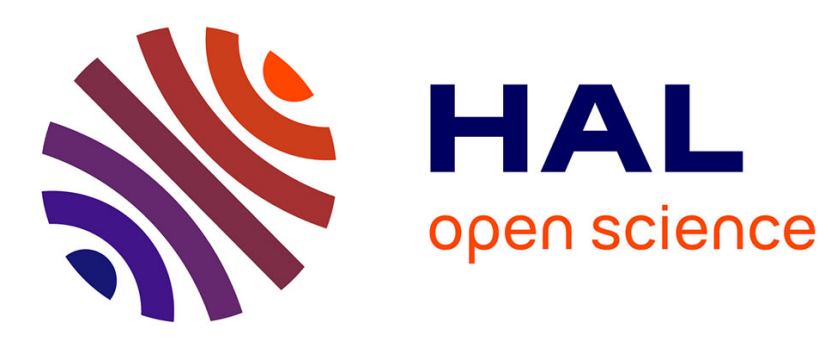

\title{
Validation factorielle d'une mesure des menaces du stéréotype en langue française
}

Caroline Desombre, Mickaël Jury, Muriel Renard, Célénie Brasselet

\section{To cite this version:}

Caroline Desombre, Mickaël Jury, Muriel Renard, Célénie Brasselet. Validation factorielle d'une mesure des menaces du stéréotype en langue française. Annee Psychologique, 2019, 120 (4), pp.251269. 10.3917/anpsy1.204.0251 . hal-03139811

\section{HAL Id: hal-03139811 https://hal.science/hal-03139811}

Submitted on 10 Nov 2021

HAL is a multi-disciplinary open access archive for the deposit and dissemination of scientific research documents, whether they are published or not. The documents may come from teaching and research institutions in France or abroad, or from public or private research centers.
L'archive ouverte pluridisciplinaire HAL, est destinée au dépôt et à la diffusion de documents scientifiques de niveau recherche, publiés ou non, émanant des établissements d'enseignement et de recherche français ou étrangers, des laboratoires publics ou privés. 


\title{
Validation factorielle d'une mesure des menaces du stéréotype en langue française ${ }^{1}$
}

\author{
Caroline Desombre ${ }^{1}$, Mickael Jury ${ }^{2}$, Muriel Renard ${ }^{3}$ et Célénie Brasselet ${ }^{1}$
}

1 Univ. Lille, ULR 4072 PSITEC Psychologie : Interactions Temps Émotions Cognition, F-59000 Lille, France caroline.desombre@inspe-lille-hdf.fr

2 INSPE Clermont Auvergne, Laboratoire ACTé - EA 4281

3 Lycée Jean Bart, Dunkerque

\section{Résumé}

Selon le modèle Multi-Menaces de Shapiro et Neuberg (2007), la menace du stéréotype serait polymorphe et dépendrait de la source de la menace (c.-à-d., qui peut utiliser ces actions comme une indication des habiletés ? Soi, les membres de l'endogroupe, ou les membres de l'exogroupe ?) et de la cible de la menace (c.-à-d., quelle performance reflète l'action ? La performance du soi ou du groupe ?). L'objectif de cet article est de proposer une validation factorielle d'un outil francophone de mesure multimenaces du stéréotype (Shapiro, 2011). Pour ce faire, 273 lycéennes et lycéens en classe de seconde, première et terminale ont répondu au questionnaire dans un domaine stéréotypique correspondant (en mathématiques pour les filles et en français pour les garçons). Les analyses factorielles confirmatoires indiquent que conformément aux propositions théoriques de Shapiro et Neuberg (2007), un modèle à six facteurs s'ajuste le mieux aux données. Ces analyses soulignent la pertinence de distinguer différents types de menace du stéréotype en fonction de la source et de la cible de la menace. Les résultats sont discutés en termes d'implications théoriques et pratiques.

Mots-clés : menace du stéréotype ; modèle multi-menaces ; source de la menace ; cible de la menace.

Une enquête récente de l'Organisation de coopération et de développement économiques (OCDE) sur le Programme international pour le suivi des acquis des élèves (PISA) a confirmé des inégalités constantes et récurrentes dans la réussite de certains groupes d'élèves (OCDE, 2016). Par exemple, il apparaît que les garçons sont moins efficients en lecture que les filles et, de manière similaire, que les filles ont des scores en mathématiques inférieurs à ceux des garçons. Dans la littérature, ces différences de performance ont notamment été interprétées au regard de l'influence de stéréotypes négatifs (Régner, Steele, \& Huguet, 2014). En effet, les élèves, en raison de la réputation négative de leur groupe dans un domaine particulier (par ex., les garçons dans les matières littéraires et les filles dans les matières scientifiques) peuvent avoir peur de confirmer les stéréotypes liés à leur groupe, être en conséquence moins efficients et, in fine, renforcer cette réputation négative (Croizet \& Claire, 1998 ; Huguet \&

\footnotetext{
${ }^{1}$ Ce papier a été accepté pour publication dans l'Année Psychologique. Cette version est la version post-print.
} 
Régner, 2009 ; Pansu et al., 2016 ; Steele \& Aronson, 1995). Ce phénomène est appelé menace du stéréotype (Steele \& Aronson, 1995).

\section{De la menace aux menaces du stéréotype}

Le phénomène de menace du stéréotype a été mis en évidence par Steele et Aronson (1995). Ces auteurs ont montré que la manière de présenter un test pouvait affecter la performance d'étudiants afro-américains. Plus précisément, ils ont montré que les étudiants afroaméricains réussissaient moins bien que des étudiants européens-américains lorsqu'un test était présenté comme une mesure de capacités verbales. À l'inverse, lorsque le test était présenté comme une tâche de résolution de problème sans évaluation des capacités verbales, les étudiants afroaméricains réussissaient tout aussi bien que les étudiants européensaméricains. Cette différence d'efficience en fonction de la présentation du test a été interprétée comme la conséquence du stéréotype de moindre intelligence associé aux personnes afro-américaines. Les travaux subséquents ont permis de montrer que la menace du stéréotype peut concerner tous les groupes, qu'ils soient stigmatisés (par ex., les étudiants en situation de handicap, Desombre, Anegmar, \& Delelis, 2018) ou non (par ex., les hommes, Leyens, Désert, Croizet, \& Darcis, 2000) et que les répercussions peuvent être diverses que ce soit sur l'efficience cognitive (par ex., dans des tâches verbales, Pansu et al., 2016), l'efficience motrice (par ex., les performances sportives, Chalabaev, Sarrazin, Stone, \& Cury, 2008) ou encore sur les intentions comportementales en lien avec les activités culturelles (Bourguignon, Desmette, Yzerbyt, \& Herman, 2007). La plupart de ces travaux ont considéré la menace du stéréotype comme unique (c.-à-d., une seule forme de menace) même si Steele reconnaissait déjà en 1997 que les menaces pouvaient varier tant sur la forme que l'origine ou même dans les situations dans lesquelles elles s'appliquent (Steele, 1997). Les travaux de Shapiro et Neuberg (2007) ont davantage formalisé ces différentes menaces et ont proposé un modèle dans lequel les craintes ressenties par les individus se différencient en fonction des sources et des cibles de la menace. Plus précisément, la source de la menace fait référence aux personnes qui, dans la situation évaluative, sont en position de juger l'individu stéréotypé négativement. Ainsi, il peut s'agir de l'individu lui-même, d'un ou plusieurs membres de son groupe d'appartenance et/ou d'un ou plusieurs membres de l'exogroupe. La cible de la menace concerne, quant à elle, l'objet du jugement, c'est-à-dire l'individu et/ou à travers l'individu, le groupe dans son ensemble. Ainsi, résultent de la combinaison de ces deux dimensions (source et cible de la menace) six types de menaces distincts (tableau I) : la menace du concept de soi (Self-Concept Threat), la menace de la réputation personnelle par l'endogroupe (Own-Reputation Threat - Ingroup), la menace de la réputation du groupe par l'endogroupe (Group-Reputation Threat - Ingroup), la menace du concept du groupe (GroupConcept Threat), la menace de la réputation personnelle par l'exogroupe (Own-Reputation Threat - Outgroup), et la menace de la réputation du groupe par l'exogroupe (GroupReputation Threat - Outgroup). 
Tableau I

Les différents types de menace selon le modèle de Shapiro et Neuberg (2007)

\begin{tabular}{lll}
\hline \multirow{2}{*}{ Source de la menace } & Soi & \multicolumn{2}{c}{ Cible de la menace } \\
\hline Soi & Menace du concept de soi & $\begin{array}{l}\text { Menace du concept du } \\
\text { groupe }\end{array}$ \\
$\begin{array}{l}\text { Membre-s du groupe } \\
\text { d'appartenance }\end{array}$ & $\begin{array}{l}\text { Menace de la réputation } \\
\text { personnelle par } \\
\text { Membre-s d'un autre groupe }\end{array}$ & $\begin{array}{l}\text { Menace de la réputation du } \\
\text { groupe par l'endogroupe }\end{array}$ \\
& $\begin{array}{l}\text { Menace de la réputation } \\
\text { personnelle par l'exogroupe }\end{array}$ & $\begin{array}{l}\text { Menace de la réputation du } \\
\text { groupe par l'exogroupe }\end{array}$ \\
\hline
\end{tabular}

Des menaces et des chutes de performances différentes

Ces différents types de menace ont été mises en évidence à travers des chutes de performances contrastées (Desombre, Jury, Bagès, \& Brasselet, 2019 ; Jamieson \& Harkin, 2010 ; Wout, Danso, Jackson, \& Spencer, 2008 ; Zhang, Schmader, \& Hall, 2013). Zhang et collaborateurs (2013, étude 2 ) ont par exemple mesuré l'effet modérateur des cibles de la menace sur les performances des étudiantes en mathématiques. Ces dernières ont été manipulées à travers la possibilité ou non de faire le lien entre l'individu et sa performance. Plus précisément, dans la condition menace de la réputation personnelle, les participant-e-s étaient amené-es à indiquer sur le test, leur véritable nom et leur sexe. Dans la condition menace de la réputation du groupe, les participant-e-s étaient invité-e-s à indiquer un nom fictif sur le test, nom pouvant être masculin ou féminin. En d'autres termes, dans cette condition, les performances ne pouvaient pas être reliées à l'individu. Les résultats montrent que les performances en mathématiques d'étudiantes chutent de manière plus importante lorsque la situation génère une menace de la réputation personnelle (ciblant l'individu) plutôt qu'une menace de la réputation du groupe (ciblant l'appartenance groupale de l'individu). Cet effet se retrouve quel que soit le genre du prénom utilisé par les participantes. C'est donc bien la possibilité de dissocier ou non l'identité personnelle de la performance qui est en jeu ici.

D'autres études (Desombre et al., 2018 ; Jamieson \& Harkin, 2010 ; Wout et al., 2008) ont mis en évidence l'effet modérateur de la source de la menace sur les performances. Wout et al. (2008) ont par exemple montré, toujours auprès de femmes, que les performances en mathématiques étaient plus impactées par une situation de menace du concept de soi (activée par l'évaluation personnelle de la performance et le caractère privé des résultats) plutôt qu'une situation de menace de la réputation du groupe (activée par l'évaluation des performances à l'échelle du groupe - c'est-à-dire comparaison du groupe des hommes et des femmes - et le caractère public des résultats). Dans la même lignée, Desombre et 
collaborateurs (2019) ont montré l'effet modérateur de la source de la menace sur la chute de performances. Les participant-e-s ont été menacé-e-s sur leur identité de valide concernant leur capacité d'attention et de concentration (en comparaison aux personnes sourdes) dans l'étude 1 et concernant leur capacité mémorielle (en comparaison aux personnes aveugles) dans l'étude 2 . Ils/elles étaient aléatoirement répartis dans une des trois conditions expérimentales : menace réduite (pas de comparaison avec des personnes sourdes ou aveugles), menace de la réputation personnelle (comparaison - potentiellement défavorable - avec des personnes sourdes ou aveugles et évaluation publique) et menace du concept de soi (comparaison - potentiellement défavorable - avec des personnes sourdes ou aveugles et évaluation privée). Les résultats indiquent que la menace du concept de soi génère davantage de chutes de performances que la menace de la réputation personnelle.

Ces divers résultats tendent à montrer que les chutes de performances sont modérées par les sources et les cibles de la menace, soutenant ainsi le modèle multi-menaces. Mais qu'en estil au niveau des perceptions de menaces?

\section{Des perceptions de menaces différentes}

Depuis la publication du modèle de Shapiro et Neuberg (2007), plusieurs études ont tenté de mettre en évidence l'existence de ces différentes formes de menace au niveau des perceptions (Berjot, 2003 ; Berjot \& Drozda-Senkowska, 2003 ; Laurin, 2017 ; Pennington, Heim, Levy, \& Larkin, 2016; Pennington, Kaye, \& McCann, 2018; Shapiro, 2011; Shapiro \& Williams, 2012 ; Zhang et al., 2013, étude 1). Par exemple, Shapiro (2011) a tenté de montrer que les menaces ressenties pouvaient être différentes d'un groupe à l'autre en fonction de différentes variables. Elle a demandé à des groupes stigmatisés sur différentes dimensions (c.à-d., leur appartenance ethnique et religieuse, leur santé mentale ou leur poids) de reporter le degré de menace perçue en fonction de la source et de la cible de la menace. Deux variables étaient prises en considération : l'identification au groupe d'appartenance et l'adhésion au stéréotype. Les résultats ont mis en évidence que les menaces perçues différaient selon les groupes et ce en fonction de l'identification au groupe d'appartenance et de l'adhésion au stéréotype. Ainsi, les individus appartenant à un groupe dont l'identification est élevée (groupes ethnique et religieux) rapportaient davantage de menace dont la cible est le groupe que les individus appartenant à un groupe dont l'identification est faible (personnes atteintes de maladie mentale et personnes en surpoids). En outre, les membres de groupe avec une forte adhésion aux stéréotypes rapportaient davantage de menace dont la source est soi que les membres de groupe avec peu d'adhésion aux stéréotypes. Cette étude a donc confirmé que les groupes sont susceptibles d'être soumis et/ou sont sensibles à des menaces différentes.

D'autres études ont montré que le contexte pouvait activer des menaces différentes. Par exemple, Larkin et Pines (2011) ont mesuré l'effet de la source de la menace sur les perceptions des filles en mathématiques à travers le contexte (public ou privé) de l'annonce 
du résultat. Dans la condition publique, les participant-e-s étaient invité-e-s à compléter de manière anonyme un questionnaire dont le résultat serait prétendument public. Dans la condition privée, les participant-e-s renseignaient le questionnaire de manière anonyme mais étaient informé-e-s qu'ils-elles seraient les seul-e-s à avoir accès à leur résultat. Les résultats indiquent que la perception de menace des participantes est différente de celle des participants uniquement dans la condition publique. En condition privée, aucune différence n'apparaît entre les participants et les participantes. Berjot (2003, voir aussi Berjot \& DrozdaSenkowska, 2003) a, quant à elle, mesuré l'effet de la cible de la menace sur les stratégies employées par les individus. Les résultats montrent que la menace du soi mène à une stratégie de mobilité sociale et la menace du groupe, à une stratégie de créativité sociale. La menace cumulée des deux sources génère, quant à elle, une stratégie de désengagement.

Ces premières études ont donc mis en évidence l'existence des différents types de menace du stéréotype, soulignant ainsi la pertinence d'une approche multi-menaces. Cependant, beaucoup de questions restent en suspens et davantage de développements sont nécessaires. En effet, dans l'état actuel de la recherche, les variables modératrices de chaque type de menace n'ont pas toutes été identifiées. Dans la même veine, les processus sous-jacents aux différents types de menace et notamment les variables médiatrices n'ont pas fait l'objet d'une réflexion approfondie. Ainsi, il est possible que les pensées intrusives par exemple (Cadinu, Maass, Rosabianca, \& Kiesner, 2005) et notamment leur contenu, puissent médiatiser différemment la chute des performances selon la source de la menace (Shapiro \& Neuberg, 2007). Ainsi, lorsque la source de la menace est l'individu, les idées intrusives devraient être centrées sur le sentiment de compétence alors que quand la menace provient des autres, celles-ci devraient concerner la peur d'être rejeté par les observateurs ou la méfiance à leur égard. À notre connaissance, la communauté francophone ne dispose pas encore d'outil pour participer à cet effort de recherche (les outils existants étant essentiellement anglophones, voir Shapiro, 2011). L'objectif de cette étude est de contribuer aux recherches sur les menaces du stéréotype en proposant un outil de mesure de ces menaces.

\section{Objectif de l'étude}

La présente étude vise à traduire et confirmer la structure factorielle d'une échelle de mesure multi-menaces du stéréotype (Shapiro, 2011). Les qualités psychométriques de cet outil utilisé par Shapiro (2011) ont fait l'objet d'une investigation minimale avec le seul calcul des intercorrélations entre les dimensions et des alphas de Cronbach. Dès lors, nous avons souhaité confirmer la structure à six dimensions de l'outil. Cet outil pourrait être utilisé dans des contextes différents avec deux objectifs. Tout d'abord, il devrait permettre de comparer les perceptions de menace entre différents groupes afin de proposer des actions ciblées en fonction des caractéristiques du groupe. En effet, les groupes - en fonction de leur statut, l'identification de ses membres, le domaine menacé, etc. - peuvent être sensibles à des menaces différentes (Shapiro, 2011). En situation évaluative, certains groupes pourraient ainsi davantage craindre le regard des autres alors que d'autres groupes pourraient craindre leur 
propre regard sur leurs performances. Mais ces menaces peuvent aussi être activées, pour un même groupe, par des situations différentes. Autrement dit, en fonction des situations sociales, c'est-à-dire la présence ou non des autres dans le contexte évaluatif, la compétition ou non entre individus, etc., les individus ressentiraient des menaces différentes. Dans cette perspective, la menace perçue ne dépendrait pas du groupe et de ses caractéristiques mais plutôt de la situation dans laquelle ses membres sont placés. Cet outil pourrait ainsi être utilisé dans des situations écologiques et/ou pour contrôler les manipulations expérimentales dans les études sur les menaces du stéréotype.

\section{Méthode}

Participants et procédure

Deux cent quatre-vingt-quatre lycéen-ne-s d'un lycée général et technologique en zone urbaine ont participé à cette étude. Onze participante-s ont dû être retirés de l'échantillon car ils ont fourni des réponses inutilisables (c.-à-d., ils ont fourni des commentaires inappropriés dans la zone prévue à cet effet, laissant de sérieux doutes sur le fait que le questionnaire ait été rempli avec rigueur). L'échantillon final était composé de 273 lycéen-ne-s (205 garçons et 68 filles) appartenant à des classes de seconde (46\%), de première $(23 \%)$ et de terminale générale (31\%), avec un âge moyen de 16.57 ans $(E T=5.16)$.

Il est important de noter que la disparité entre le nombre de filles et de garçons dans cette étude (c.-à-d., 1/4 de filles) est représentative de la disparité en termes de répartition générale au sein de ce lycée (13\% des élèves de l'effectif total sont des filles). En effet, ce lycée dispense un grand nombre de formations technologiques et professionnelles vers lesquelles les garçons s'orientent et sont orientés plus favorablement que les filles. Le choix de cet établissement est donc justifié par le fait que cette asymétrie induirait des difficultés comme par exemple les tensions entre garçons et filles observées par l'équipe enseignante; ce contexte rendrait ainsi fortement saillants les stéréotypes de genre.

Avant la passation des questionnaires, le chef d'établissement et les parents ont donné leur accord. Les participant-e-s étaient invité-e-s à remplir un questionnaire en ligne sur un temps de classe. Ils-elles complétaient tout d'abord un formulaire de consentement concernant leur participation à l'étude ; aucun refus n'a été constaté. Les participant-e-s commençaient par indiquer leur sexe. Selon leur réponse, ils-elles étaient alors orienté-e-s vers le questionnaire mesurant leur perception de menace dans le domaine stéréotypique correspondant (c.-à-d., en mathématiques pour les filles et en français pour les garçons). Pour réinstaller le contexte de classe correspondant au domaine, une question introductive demandant de rappeler le plus d'informations possibles sur le dernier cours dans ce domaine leur était posée (c.-à-d., " Ici nous vous demandons de vous rappeler le dernier cours de [Mathématiques/Français] auquel vous avez assisté et de rappeler, ci-dessous, un maximum de détails concernant le déroulé de celui-ci (ex: nom du professeur, heure de la journée, ressenti, éléments étudiés...) 
»). À la suite de cette première question, les participant-e-s devaient répondre au questionnaire sur les différents types de menace. Enfin, les participant-e-s complétaient des informations démographiques (par ex., âge, classe).

Il est à noter que cette étude incluait d'autres mesures dans le cadre d'un projet plus large de recherche. Néanmoins, ces mesures n'étant pas exploitées ici, celles-ci ne seront pas présentées.

\section{Matériel}

L'outil proposé par Shapiro pour mesurer les différentes menaces dans un domaine stéréotypique (voir Étude 1, Shapiro, 2011 ; voir aussi Shapiro \& Neuberg, 2007) a été utilisé ici. Les items ont été traduits de l'anglais au français par un comité d'experts et adaptés à la population lycéenne. Dans son étude, Shapiro (2011, étude 1) a utilisé un questionnaire de 12 items permettant de mesurer la menace du concept de soi, la menace du concept de groupe, la menace de la réputation personnelle et la menace de la réputation du groupe. Pour ces deux dernières menaces, l'auteure n'a pas dissocié la source (endogroupe ou exogroupe) ; le terme générique "les autres " a été utilisé. Nous souhaitions pouvoir tester le modèle dans sa globalité. C'est la raison pour laquelle nous avons introduit cette variable en spécifiant précisément la source de la menace. Un questionnaire de 18 items ( 3 items pour chaque type de menace) en résulte.

Les participant-e-s devaient exprimer leur degré d'accord ou de désaccord avec ces items sur une échelle de Likert en 5 points allant de 1, Pas du tout d'accord à 5, Tout à fait d'accord. Trois items mesuraient la menace du concept de soi (par ex., "Parfois dans mes cours de [français/mathématique], j'ai peur que mon comportement m'amène à penser que je suis moins [bon/bonne] que les [filles/garçons] »), 3 mesuraient la menace de la réputation personnelle par l'endogroupe (par ex., "Parfois dans mes cours de [français/mathématique], j'ai peur que mon comportement puisse prouver aux autres [garçons/filles] que je suis moins [bon/bonne] en [français/mathématique] que les [filles/garçons] ».), 3 mesuraient la menace de la réputation du groupe par l'endogroupe (par ex., "Parfois dans mes cours de [français/mathématique], j'ai peur que mon comportement puisse prouver aux autres [garçons/filles] que les [garçons/filles] sont moins [bons/bonnes] en [français/mathématique] que les [filles/garçons] ».), 3 mesuraient la menace du concept du groupe (par ex., "Parfois dans mes cours de [français/mathématique], j'ai peur que mon comportement me prouve que les [garçons/filles] sont moins [bons/bonnes] en [français/mathématique] que les [filles/garçons] ».), 3 mesuraient la menace de la réputation personnelle par l'exogroupe (par ex., "Parfois dans mes cours de [français/mathématique], j'ai peur que mon comportement amène les [filles/garçons] à se comporter différemment avec moi parce que je suis [un garçon/une fille]. ».), et 3 mesuraient la menace de la réputation du groupe par l'exogroupe (par ex., "Parfois dans mes cours de [français/mathématique], j'ai peur que mon comportement amène les [filles/garçons] à voir négativement le groupe des [garçons/filles].»). 
II est à noter que les participant-e-s répondaient par bloc d'items (c.-à-d., un bloc était équivalent à un type de menace) dont l'ordre était aléatoire, blocs au sein desquels l'ordre des items était également aléatoire. La liste complète des items est présentée dans le Tableau II.

La traduction de l'outil de Shapiro (2011) s'est faite en plusieurs étapes. La première étape a consisté à une première traduction en français de l'outil en anglais. Pour cette première version, deux enseignants chercheurs en psychologie, impliqués dans la recherche, ont traduit, confronté et discuté les items traduits. Après cette version préliminaire de l'outil, un des enseignants de la première phase et une experte en psychologie extérieure à la recherche ont été sollicités pour évaluer cette première version et vérifier à la fois l'intelligibilité et la validité du contenu des items. Quelques modifications de forme ont été réalisées. Dans une troisième phase, cinq collégiens de $6^{\mathrm{e}}$ (plus jeunes que la population cible) ont été sollicités pour vérifier la clarté des items. Aucune modification n'a été apportée à la suite de leur lecture.

\section{Résultats}

Afin de vérifier la validité conceptuelle de l'outil, des analyses factorielles confirmatoires ont été réalisées à l'aide du paquet «lavaan» (Rosseel, 2012) pour le logiciel R (R Core Team, 2014). Les analyses ont été réalisées sur la matrice de covariance et la solution a été générée sur la base d'un estimateur robuste du maximum de vraisemblance. Les variables étaient standardisées et les données manquantes ont été gérées par la méthode FIML (c.-à-d., Full Information Maximum Likelihood, Enders, 2006). Comme de coutume dans la littérature (pour des recommandations, voir Jackson, Gillapsy, \& Purc-Stephenson, 2009), nous avons utilisé plusieurs indicateurs afin d'évaluer l'ajustement du modèle aux données, le CFI (c.-à-d., le Comparative Fit Index), le RMSEA (c.-àd., le Root-Mean-Square Error of Approximation) et le $S R M R$ (c.-à-d., le Standardized Root Mean Square Residual).

Ainsi, sur la base de la théorie et de la précédente étude (Shapiro, 2011), un modèle à six facteurs (c.-à-d., " Menace du concept de Soi », " Menace de la réputation personnelle par l'endogroupe ", "Menace de la réputation du groupe par l'endogroupe ", "Menace du concept de Groupe ", "Menace de la réputation personnelle par l'exogroupe ", et " Menace de la réputation du groupe par l'exogroupe ") était testé. Les résultats traduisent, conformément à l'hypothèse, que ce modèle s'ajuste bien aux données. En effet, les critères de satisfaction concernant l'ensemble des indicateurs ont été atteints, CFI =.95, RMSEA = $.051,90 \% \mathrm{Cls}[.042 ; .060], \mathrm{SRMR}=.033$, ce, en dépit d'un chi-carré significatif, $\chi^{2}(120, \mathrm{~N}=$ $273)=205.47, p=.001$. Il est à noter que le poids de saturation à l'intérieur de chaque facteur est satisfaisant, de même que la cohérence interne pour chaque type de menace (voir Tableau II). Les statistiques descriptives et les corrélations entre les facteurs sont présentées dans le Tableau III. 
Tableau II

Liste des items pour le questionnaire mesurant les différents types de menace dans un domaine stéréotypique (exemple : les garçons en Français)

\begin{tabular}{lll}
\hline Facteurs & Items & $\begin{array}{l}\text { Poids de } \\
\text { saturation }\end{array}$ \\
\hline
\end{tabular}

Parfois, dans mes cours de français, j'ai peur que

mon comportement...

Menace du concept

de soi

...m'amène à penser que je ne suis pas bon en français

...m'amène à penser que je suis moins bon en

français que les filles

...me confirme que je suis moins bon en français

que les filles

Menace du concept

de groupe

...m'amène à accepter l'idée que les garçons

sont moins bons en français que les filles

...me prouve que les garçons sont moins bons en

français que les filles

.93

...m'amène à penser que les garçons sont moins

bons en français que les filles

Réputation personnelle

(endogroupe)

...pousse les autres garçons à me juger

négativement parce que les garçons ont la

réputation d'être moins bons en français que les

filles

...puisse prouver aux autres garçons que je suis

moins bon en français que les filles

...pousse les autres garçons à se comporter

différemment avec moi parce que je suis un

garçon

Réputation personnelle

(exogroupe)

... amène les filles à me juger négativement

parce que les garçons ont la réputation d'être

moins bons en français

... amène les filles à me voir négativement parce

que je suis un garçon

... amène les filles à se comporter différemment

avec moi parce que je suis un garçon 
Réputation du groupe

(endogroupe)

...puisse prouver aux autres garçons que les

garçons sont moins bons en français que les

filles

...pousse les autres garçons à se représenter négativement le groupe des garçons

...renforce l'idée dans la tête des autres garçons

que les garçons sont moins bons en français que les filles

Réputation du groupe

(exogroupe)

... renforce dans la tête des filles l'idée que les

garçons sont moins bons en français que les

filles

... prouve aux filles que les garçons sont moins

bons en français que les filles

... amène les filles à voir négativement le groupe 
Tableau III

Statistiques descriptives et corrélations

\begin{tabular}{|c|c|c|c|c|c|c|c|}
\hline & $M$ & $E T$ & 1 & 2 & 3 & 4 & 5 \\
\hline $\begin{array}{l}\text { 1. Menace du } \\
\text { concept de soi }\end{array}$ & 2.02 & 0.98 & - & & & & \\
\hline $\begin{array}{l}\text { 2. Menace du } \\
\text { concept du } \\
\text { groupe }\end{array}$ & 1.76 & 0.92 & $.68^{*}$ & - & & & \\
\hline $\begin{array}{l}\text { 3. Réputation } \\
\text { personnelle } \\
\text { (endogroupe) }\end{array}$ & 1.73 & 0.85 & $.62^{*}$ & $.68^{*}$ & - & & \\
\hline $\begin{array}{l}\text { 4. Réputation } \\
\text { personnelle } \\
\text { (exogroupe) }\end{array}$ & 1.80 & 0.92 & $.59^{*}$ & $.75^{*}$ & $.68^{*}$ & - & \\
\hline $\begin{array}{l}\text { 5. Réputation du } \\
\text { groupe } \\
\text { (endogroupe) }\end{array}$ & 1.89 & 0.97 & $.62^{*}$ & $.71^{*}$ & $.72^{*}$ & $.68^{*}$ & - \\
\hline $\begin{array}{l}\text { 6. Réputation du } \\
\text { groupe } \\
\text { (exogroupe) }\end{array}$ & 1.85 & 0.98 & $.61^{*}$ & $.78^{*}$ & $.70^{*}$ & $.81^{*}$ & $.77^{*}$ \\
\hline
\end{tabular}

Note : ${ }^{*} p<.001$

Afin de s'assurer que ce modèle était celui qui s'ajustait le mieux aux données, ce dernier a été comparé à 4 autres modèles. Ainsi, ce modèle à six facteurs a été comparé à (1) un modèle « 2 » où les types de menaces étaient indifférenciés (c.-à-d., un seul facteur), (2) un modèle " 3 » où seules les sources de la menace étaient distinguées (c.-à-d., trois facteurs, un facteur « Menace par le Soi ", un facteur "Menace par l'endogroupe ", et un facteur "Menace par l'exogroupe "), (3) un modèle " 4 " où seules les cibles étaient distinguées (c.-à-d., deux facteurs, un facteur " Menace de l'individu » et un facteur " Menace du groupe »), et (4) un modèle " 5 " dans lequel les sources de la menace générée par les autres (endogroupe et exogroupe) étaient confondues (c.-à-d., quatre facteurs, un facteur « Menace du concept de Soi ", un facteur "Menace du concept du Groupe ", un facteur "Menace de la réputation personnelle » et un facteur " Menace de la réputation du groupe »). Les analyses ont révélé que le modèle à six facteurs s'ajustait mieux aux données que le modèle $2, \Delta \chi^{2}=165.79, p<$ .001 , le modèle $3, \Delta \chi^{2}=120,23, p<.001$, le modèle $4, \Delta \chi^{2}=130.53, p<.001$, et le modèle 5 , $\Delta \chi^{2}=50.08, p<.001$.

À titre exploratoire, nous avons comparé les menaces ressenties en fonction du sexe (voir tableau IV). Les résultats révèlent que les perceptions des garçons et les filles ne diffèrent significativement que sur la menace du concept de groupe, $t(143,15)=-2,30, p=0,023$. Ainsi, 
il semble que les garçons $(M=1,83, E T=1,67)$ fassent davantage l'expérience d'une menace du concept du groupe que les filles $(M=1,56, E T=1,00)$.

Tableau IV.

Différences de moyennes en fonction du sexe des participants.

\begin{tabular}{|c|c|c|c|c|c|}
\hline & Sexe & $M$ & $E T$ & $t_{\text {Welch }}$ & $p$ \\
\hline \multirow[t]{3}{*}{ 1. Menace du concept de soi } & Filles & 2,05 & 0,99 & \multirow{3}{*}{0,33} & \multirow{3}{*}{0,74} \\
\hline & & & & & \\
\hline & Garçons & 2,00 & 0,97 & & \\
\hline \multirow[t]{3}{*}{ 2. Menace du concept du groupe } & Filles & 1,56 & 1,00 & \multirow{3}{*}{$-2,30$} & \multirow{3}{*}{0,023} \\
\hline & & & & & \\
\hline & Garçons & 1,83 & 1,67 & & \\
\hline \multirow[t]{3}{*}{ 3. Réputation personnelle (endogroupe) } & Filles & 1,63 & 0,83 & \multirow{3}{*}{$-1,07$} & \multirow{3}{*}{0,29} \\
\hline & & & & & \\
\hline & Garçons & 1,76 & 0,85 & & \\
\hline \multirow[t]{3}{*}{ 4. Réputation personnelle (exogroupe) } & Filles & 2,01 & 1,03 & \multirow{3}{*}{1,07} & \multirow{3}{*}{0,29} \\
\hline & & & & & \\
\hline & Garçons & 1,85 & 0,95 & & \\
\hline \multirow[t]{3}{*}{ 5. Réputation du groupe (endogroupe) } & Filles & 1,72 & 0,88 & \multirow{3}{*}{$-0,94$} & \multirow{3}{*}{0,35} \\
\hline & & & & & \\
\hline & Garçons & 1,83 & 0,94 & & \\
\hline \multirow[t]{3}{*}{ 6. Réputation du groupe (exogroupe) } & Filles & 1,81 & 0,95 & \multirow{3}{*}{$-0,39$} & \multirow{3}{*}{0,70} \\
\hline & & & & & \\
\hline & Garçons & 1,87 & 1,00 & & \\
\hline
\end{tabular}

\section{Discussion}

Les recherches sur la menace du stéréotype sont particulièrement nombreuses (pour une revue récente voir Pennington et al., 2016). Néanmoins, la plupart de ces travaux ont considéré la menace du stéréotype comme une unique forme de menace alors qu'il apparaît que l'individu pourrait en réalité faire face à différentes formes de menace (Steele, 1997). Ainsi, Shapiro et Neuberg (2007) ont proposé un modèle multi-menaces dans lequel ils distinguent six différents types de menace en fonction de la source (c.-à-d., soi, les membres de l'endogroupe ou les membres de l'exogroupe) et de la cible de la menace (c.-à-d., soi ou le groupe). La distinction entre ces différentes formes de menace est signifiante puisque cellesci peuvent avoir des conséquences particulières, notamment sur les performances des individus (Desombre et al., 2019 ; Wout et al., 2008 ; Zhang et al., 2013). Ainsi, l'objectif de cette étude était de traduire en français un outil proposé dans la littérature anglophone 
(Shapiro, 2011) et de tester sa structure factorielle ; la communauté francophone dispose ainsi d'une mesure des différents types de menace du stéréotype auquel un individu peut faire face.

Globalement, les analyses réalisées sur les données récoltées auprès de lycéen-ne-s semblent indiquer que cet outil présente des qualités psychométriques satisfaisantes (structure factorielle et consistance interne). En effet, il apparaît que le modèle à six facteurs s'ajuste bien aux données, que celui-ci est même celui qui s'y ajuste le mieux (comparativement à des modèles alternatifs) et que les analyses de cohérence interne sont satisfaisantes. II semble donc que cet outil pourrait permettre d'identifier adéquatement les différentes menaces perçues par un individu selon la source et la cible de celles-ci. À ce titre, cet outil pourrait donc se révéler particulièrement utile pour les chercheur-euse-s de la communauté francophone qui souhaiteraient contribuer au débat scientifique sur les conséquences des menaces du stéréotype.

D'un point de vue théorique, outre les avantages cités précédemment, la mesure de ces différentes formes de menace pourrait permettre de comprendre certains résultats discordants de la littérature scientifique. Par exemple, lorsque l'on s'intéresse à l'effet modérateur de certaines variables sur les conséquences de la menace (pour des métaanalyses, voir Doyle \& Voyer, 2016 ; Flore \& Witcherts, 2015 ; Lamont, Swift, \& Abrams, 2015 ; Nguyen \& Ryan, 2008 ; Picho, Rodriguez, \& Finnie, 2013), il n'est pas rare de constater des résultats incohérents. Ces incohérences pourraient être liées au fait que les modérateurs identifiés sont en fait spécifiques à certaines formes de menaces. Par exemple, selon le modèle multi-menaces (Shapiro, 2011 ; Shapiro \& Neuberg, 2007), I'identification au groupe (un modérateur connu de l'effet de la menace du stéréotype, Schmader, 2002) devrait modérer les menaces ciblant le groupe mais pas les menaces ciblant l'individu. Wout et collaborateurs (2008) ont confirmé cette hypothèse en montrant que, dans le domaine mathématique, l'identification au groupe modère la performance de femmes en situation de menace de la réputation du groupe ( $c^{\prime}$ est-à-dire ciblant le groupe) mais pas la performance de femmes en situation de menace du concept de soi (c'està-dire ciblant l'individu). Dans le même ordre d'idées, Zhang et collaborateurs (2013) ont montré que l'identification au domaine des mathématiques (un autre modérateur connu de l'effet de menace, Appel, Kronberger, \& Aronson, 2011) modère les menaces centrées sur l'individu mais pas les menaces centrées sur le groupe. La disponibilité d'un outil francophone de mesure des menaces du stéréotype permettra donc également aux chercheur-euse-s de cette communauté de contribuer à la compréhension plus précise des phénomènes de menace du stéréotype.

Cet outil présente néanmoins certaines limites qu'il faut mentionner. Tout d'abord, cette étude a été réalisée dans un contexte spécifique puisque nous avons interrogé des adolescente-s (dont l'identité sociale est en construction) en contexte scolaire. Une nouvelle étude devrait être réalisée dans un contexte extra-scolaire, auprès d'une population plus diverse, 
afin de tester la généralisation potentielle de l'outil et répliquer les résultats antérieurs (Shapiro, 2011). Dans le même ordre d'idées, l'établissement choisi, majoritairement composé de garçons, pose la question de la généralisation des résultats. Par ailleurs, en regardant le Tableau III, il est possible de s'interroger sur l'ampleur de la menace ressentie par les participant-e-s. En effet, il apparaît que les scores moyens de menace sont faibles malgré un contexte scolaire hautement évaluatif. Une étude expérimentale dans laquelle les différents types de menace seraient manipulés pourrait permettre de s'assurer de la sensibilité de cet outil de mesure. Le fait que les adolescent-e-s ont vécu de manière limitée des expériences de discrimination pose également question quant à la généralisation de nos résultats. II serait intéressant de soumettre cet outil à des groupes qui ont vécu davantage de situations discriminatoires pour en mesurer les effets. En outre, en raison du niveau très élevé de corrélation entre les différentes formes de menaces (c.-à-d., .59<= $r<=.81$ ), il faut reconnaître que la question de la distinction des différents types de menace pourrait être posée. Néanmoins, le fait que la structure en six facteurs soit la mieux ajustée aux données (comparativement à des modèles ne faisant pas la distinction entre les menaces) tend plutôt à renforcer l'hypothèse de l'existence de formes de menaces distinctes même si fortement reliées. Finalement, en lien avec la question de la distinction des types de menaces, il est important de noter que les items du questionnaire sont parfois très proches sémantiquement et qu'ils ne sont peut-être pas toujours compréhensibles par de jeunes participants. En cela, I'utilisation de cet outil semble être limitée.

En dépit de ces limites, cet outil ouvre des perspectives d'un point de vue pratique. En effet, celui-ci devrait permettre de proposer, notamment dans l'institution scolaire, des dispositifs de lutte contre les effets de la menace du stéréotype plus adaptés. En effet, savoir que les filles, confrontées à une réputation négative en mathématiques, sont plus sensibles à certaines menaces plutôt qu'à d'autres permet d'envisager des actions de remédiation plus ciblées. Par exemple, si les filles craignent davantage leur propre regard sur leur performance en mathématiques plutôt que celui des autres, comme c'est le cas dans cette étude, un travail de déconstruction des stéréotypes orientés vers les filles sera plus efficace qu'un travail sur leur conscience de la stigmatisation ou sur les stéréotypes des garçons à l'égard des filles. Le même raisonnement pour les garçons ou pour tout autre groupe stéréotypé négativement dans l'institution scolaire pourrait s'appliquer. D'une manière générale, une connaissance plus fine des phénomènes auxquels sont confrontés les individus permet une prise en charge d'autant mieux ajustée. 


\section{Références}

Appel, M., Kronberger, N., \& Aronson, J. (2011). Stereotype threat impedes ability building: Effects on test preparation among women in science and technology. European Journal of Social Psychology, 41, 904913. doi: 10.1002/ejsp.835

Berjot, S. (2003). Qui dit que je ne suis pas intelligent ? Stratégies de mobilité individuelle et de créativité sociale chez les cibles stigmatisées selon la saillance du contexte menaçant : personnel ou social. Nouvelle Revue de Psychologie Sociale, 3, 297-309.

Berjot, S., \& Drozda-Senkowska, E. (2003). Stratégies d'infirmation du stéréotype et performances des cibles stigmatisées [Stereotype disconfirmation strategies and performance of stigmatized targets]. Cahiers Internationaux de Psychologie Sociale, 59, 7-21.

Bourguignon, D., Desmette, D., Yzerbyt, V., \& Herman, G. (2007). Activation du stéréotype, performance intellectuelle et intentions d'action : Le cas des personnes sans emploi. Revue Internationale de Psychologie Sociale, 20, 123-153.

Cadinu, M., Maass, A., Rosabianca, A., \& Kiesner, J. (2005). Why do women underperform under stereotype threat? Evidence for the role of negative thinking. Psychological Science, 16, 572-578. doi: 10.1111/ j.0956-7976.2005.01577.x

Chalabaev, A., Sarrazin, P., Stone, J., \& Cury, F. (2008). Do achievement goals mediate stereotype threat? An investigation on females' soccer performance. Journal of Sport and Exercise Psychology, 30(2), 143158. doi: 10.1123/jsep.30.2.143

Croizet, J.-C., \& Claire, T. (1998). Extending the concept of stereotype threat to social class: The intellectual underperformance of students from low socioeconomic backgrounds. Personality and Social Psychology Bulletin, 24(6), 588-594. doi: 10.1177/0146167298246003

Desombre, C., Anegmar, S., \& Delelis, G. (2018). Stereotype threat among students with disabilities: The importance of the evaluative context on their cognitive performance. European Journal of Psychology of Education, 33, 201-214. doi: 10.1007/ s10212-016-0327-4

Desombre, C., Jury, M., Bagès, C., \& Brasselet, C. (2019). The Distinct Effect of Multiple Sources of Stereotype Threat. The Journal of Social Psychology, 159(5), 628641. doi: 10.1080/00224545.2018.1544540

Doyle, R. A., \& Voyer, D. (2016). Stereotype manipulation effects on math and spatial test performance: A meta-analysis. Learning and Individual Differences, 47, 103-116. doi: 10.1016/j.lindif.2015.12.018 
Enders, C. K. (2006). A primer on the use of modern missing-data methods in psychosomatic medicine research. Psychosomatic Medicine, 68(3), 427-436. doi:

10.1097/01.psy.0000221275.75056.d8

Flore, P. C., \& Wicherts, J. M. (2015). Does stereotype threat influence performance of girls in stereotyped domains? A meta-analysis. Journal of School Psychology, 53(1), 25-44. doi: 10.1016/j.jsp.2014.10.002

Huguet, P., \& Régner, I. (2009). Counterstereotypic beliefs in math do not protect school girls from stereotype threat. Journal

of Experimental Social Psychology, 45(4), 1024-1027. doi: 10.1016/j.jesp.2009.04.029

Jackson, D. L., Gillaspy, J. A., \& Purc-Stephenson, R. (2009). Reporting practices in confirmatory factor analysis: An overview and some recommendations. Psychological Methods, 14(1), 6-23. doi: 10.1037/ a0014694

Jamieson, J. P., \& Harkins, S. G. (2010). Evaluation is necessary to produce stereotype threat performance effects. Social Influence, 5(2), 75-86. doi: 10.1080/ 15534510903512409

Lamont, R. A., Swift, H. J., \& Abrams, D. (2015). A review and meta-analysis of agebased stereotype threat: Negative stereotypes, not facts, do the damage. Psychology and Aging, 30(1), 180-193. doi: 10.1037/ a0038586

Larkin, J. E., \& Pines, H. A. (2011). Anticipating public performance: Do women fear appearing intellectually less able? Journal of Applied Social Psychology, 41(3), 682-698. doi:10.1111/j.1559-1816.2011.00732.x

Laurin, R. (2017). Group and individual stereotype threat and identity management strategies: An investigation of rugby women. Journal of Applied Social Psychology, 47(10), 584-588. doi: 10.1111/jasp.12466

Leyens, J. P., Désert, M., Croizet, J. C., \& Darcis, C. (2000). Stereotype threat: Are lower status and history of stigmatization preconditions of stereotype threat? Personality and Social Psychology Bulletin, 26(10), 1189-1199. doi: 10.1177/0146167200262002

Nguyen, H.-H. D., \& Ryan, A. M. (2008). Does stereotype threat affect test performance of minorities and women? A metaanalysis of experimental evidence. Journal of Applied Psychology, 93(6), 1314-1334. doi: 10.1037/a0012702

OECD (2016), PISA 2015 Results (Volume I): Excellence and Equity in Education, PISA, OECD Publishing, Paris. doi: 10.1787/9789264266490-en 
Pansu, P., Régner, I., Max, S., Colé, P., Nezlek, J. B., \& Huguet, P. (2016). A burden for the boys: Evidence of stereotype threat in boys' reading performance. Journal of Experimental Social Psychology, 65, 26-30. doi: 10.1016/ j.jesp.2016.02.008

Pennington, C. R., Heim, D., Levy, A. R., \& Larkin, D. T. (2016). Twenty years of stereotype threat research: A review of psychological mediators. PLOS ONE, 11(1), e0146487. doi: 10.1371/journal.pone.0146487

Pennington, C. R., Kaye, L. K., \& McCann, J. J. (2018). Applying the multi-threat framework of stereotype threat in the context of digital gaming. PloS one, 13(2), e0192137. doi:

10.31234/osf.io/3q9tz

Picho, K., Rodriguez, A., \& Finnie, L. (2013). Exploring the moderating role of context on the mathematics performance of females under stereotype threat: A meta-analysis. The Journal of Social Psychology, 153(3), 299-333. doi: 10.1080/00224545.2012.737380

R Core Team. (2014). R: A language and environment for statistical computing. R Foundation for computing, Vienna, Austria. http: // www.R-Project.org

Régner, I., Steele, J. R., \& Huguet, P. (2014). Stereotype threat in children: Past and present. International Review of Social Psychology, 27(3), 5-12.

Rosseel, Y. (2012). lavaan: An R package for structural equation modeling. Journal of Statistical Software, 48(2), 1-36. URL: http://www.jstatsoft.org/v48/i02/

Schmader, T. (2002). Gender identification moderates stereotype threat effects on women's math performance. Journal of

Experimental Social Psychology, 38(2), 194201. doi: 10.1006/jesp.2001.1500

Shapiro, J. R. (2011). Different groups, different threats: A multi-threat approach to the experience of stereotype threats. Personality and Social Psychology Bulletin, 37, 464-480. doi:10.1177/0146167211398140

Shapiro, J. R., \& Neuberg, S. L. (2007). From stereotype threat to stereotype threats: Implications of a multi-threat framework for causes, moderators, mediators, consequences, and interventions. Personality and Social Psychology Review, 11(2), 107-130. doi: $10.1177 / 1088868306294790$

Shapiro, J. R., \& Williams, A. M. (2012). The role of stereotype threats in undermining girls' and women's performance and interest in STEM fields. Sex Roles, 66, 175183. doi:10.1007/s11199-011-0051-0 
Steele, C. M. (1997). A threat in the air: How stereotypes shape intellectual identity and performance. American Psychologist, 52(6), 613-629. doi: 10.1037/0003-066X.52.6.613

Steele, C. M., \& Aronson, J. (1995). Stereotype threat and the intellectual test performance of African Americans. Journal of Personality and Social Psychology, 69(5), 797-811. doi: 10.1037/0022-3514.69.5.797

Wout, D., Danso, H., Jackson, J., \& Spencer, S. (2008). The many faces of stereotype threat: Group-and self-threat. Journal of Experimental Social Psychology, 44(3), 792799. doi: 10.1016/j.jesp.2007.07.005

Zhang, S., Schmader, T., \& Hall, W. M. (2013). L'eggo my ego: Reducing the gender gap in math by unlinking the self from performance. Self and Identity, 12(4), 400-412. doi: $10.1080 / 15298868.2012 .687012$ 